\title{
L'homme et la bête : chiens et politique dans Les Mohicans de Paris
}

\section{Nicolas GAUTHIER, Université de Waterloo}

Le roman-feuilleton du dix-neuvième siècle est féru d'animalisation et le lecteur y rencontre fréquemment des héros aux yeux de lynx, des femmes fatales à la langue de vipère, des criminels rusés comme des singes. Ce procédé, particulièrement employé dans le portrait des acteurs du crime, s'impose d'ailleurs comme une composante constitutive de la fictionnalisation du motif de l'enquête dans le récit policier qui se développe dans la seconde moitié du dixneuvième siècle (Vareille «Préhistoire », 27 ; Homme, 45). Certains auteurs ont toutefois la main lourde, comme Alexandre Dumas père dans Les Mohicans de Paris lorsqu'il décrit le policier Jackal. Tout en se conformant aux conventions de l'époque - selon lesquelles «le policier constitue [...] un personnage honni, méprisé, récusé » (Kalifa 88) -, Jackal se singularise néanmoins en ce que son nom, qui désigne en anglais le chacal, n'est que le début du processus d'animalisation dont il fait l'objet. Après l'avoir associé «à toutes les familles de l'ordre des carnassiers digitigrades » (248), le narrateur le décrit ainsi :

[L]a chevelure, ou la crinière, ou le pelage, comme on voudra, était d'un fauve grisâtre ; les oreilles, longues, dressées contre la tête, pointues et garnies de poils, ressemblaient à celles de l'once; les yeux, d'un iris jaune le soir, vert le jour, tenaient à la fois de l'œil du lynx et du loup ; la pupille, allongée verticalement, et pareille à celle du chat, se contractait et se dilatait selon le degré d'obscurité ou de lumière dans lequel elle opérait; le nez et le menton, le museau, voulons-nous dire, était effilé comme celui d'un lévrier. Une tête de renard et un corps de putois. (248)

Le résultat de ce collage animalier n'est pas aussi informe que le célèbre chapeau de Charles Bovary mais s'approche beaucoup du registre parodique, bien que le personnage soit mis en scène d'une façon tout à fait sérieuse. Le caractère excessif du procédé (Jackal est ainsi comparé à huit animaux en quelques lignes) marque plutôt l'ironie dont fait preuve l'auteur des Mohicans de Paris face aux procédés constamment employés par certains confrères feuilletonistes. Il souligne aussi la place significative accordée au règne animal dans ce roman.

Loin de constituer ici un procédé convenu employé mécaniquement, le recours à l'animalisation dans Les Mohicans de Paris est l'espace d'une véritable réflexion sur les rapports entre l'humain et l'animal. Ceci apparaît de façon nette dans la fictionnalisation du chien, la 
figure animale la plus foisonnante chez Dumas qui a affirmé être incapable de ne pas y avoir recours (Grivel 246). Omniprésente, elle se révèle néanmoins énigmatique et ambivalente (240). Les Mohicans de Paris ne font pas exception à cette tendance puisque les différents personnages canins sont très présents mais sont associés à des types convenus et sont caractérisés par leur capacité à communiquer. Ceci induit un brouillage des limites séparant humain et animal, brouillage qui a pour fonction de permettre l'articulation des personnages canins aux enjeux fondamentaux du roman. De façon plus précise, nous observerons ici que la mise en scène du «meilleur ami de l'homme » sert à redoubler le projet politique au cœur des Mohicans de Paris.

\section{Tous les étages de la société}

Plus long roman d'Alexandre Dumas (337 chapitres, plus de 2650 pages), Les Mohicans de Paris a été écrit en collaboration avec Paul Bocage (1822-1887) et a été publié en romanfeuilleton dans Le Mousquetaire puis Le Monte-Cristo, deux journaux fondés et dirigés par Dumas. Sa publication s'est étirée sur plus de cinq années, du 25 mai 1854 au 28 juillet 1859, et a été ponctuée de plusieurs interruptions, dont une d'une année entière entre le 26 mars 1856 et le 23 avril 1857. Avec ce «roman-somme» (Dumasy 212), Dumas cherche à renouer avec sa gloire passée de feuilletoniste en reprenant le modèle proposé par Eugène Sue qui a bouleversé le roman français avec ses célèbres Mystères de Paris (1842-1843). Dans Les Mohicans de Paris s'enchevêtrent sept récits distincts qui ont pour dénominateur commun le personnage de Salvator, surhomme lumineux qui remplit bien le programme annoncé par son nom qui signifie «sauveur » en latin. Ces récits sont fondés sur des intrigues policières, amoureuses ou de mœurs tout en étant inscrits dans la trame historique des bouleversements sociopolitiques de la fin de la Restauration.

Histoire et politique, sphère privée et sphère publique, amour et économie, se côtoient donc continuellement dans cette fresque qui a pour but

de faire grimper et descendre avec nous au lecteur tous les étages de la société, depuis le pape Léon XII, auquel nous allons avoir affaire tout à l'heure, jusqu'au ravageur de ruisseaux Croc-en-Jambe [un chiffonnier]; depuis le roi Charles $\mathrm{X}$ jusqu'au tueur de chats La Gibelotte ; mais encore de faire, de temps en temps, des excursions dans les mondes inférieurs réservés aux animaux. (Dumas 1 688)

L'inclusion du règne animal est explicitement posée au moyen de la formule «mondes inférieurs » que Dumas transforme ensuite en «nos frères inférieurs » (1 688). Il souligne qu'il 
cite alors Jules Michelet qui a désigné ainsi les animaux en 1846 dans son ouvrage Le peuple (228). Nous verrons plus loin que ce n'est pas le seul point de rapprochement entre cet historien incontournable du dix-neuvième siècle et l'auteur des Mohicans de Paris. Parmi tous les animaux, le chien occupe une place particulière dans le roman : seize chapitres le mettent en vedette et lui attribuent un rôle primordial dans l'intrigue. Il n'est évidemment pas fortuit que le chien, « héros du repérage » selon la formule de Charles Grivel (248), fasse l'objet d'un tel investissement dans un roman fondé sur la recherche du savoir.

Dumas, de toute évidence, n'est pas le seul romancier à mettre en scène le chien dans la seconde moitié du dix-neuvième siècle. Offrir un tableau exhaustif de la présence romanesque de cet animal durant cette période serait une tâche titanesque. Soulignons toutefois que Dumas se distingue par sa façon de le mettre en scène. De nombreux auteurs l'intègrent dans leur récit en proposant des chiens intelligents et fidèles qui participent aux péripéties en protégeant et en aidant les héros. Plusieurs personnages canins de Jules Verne viennent à l'esprit, comme Duk dans Les aventures du capitaine Hatteras (1864), le tout premier roman des Voyages extraordinaires, ou Top dans L'ïle mystérieuse (1874-75). Cette approche sera souvent reprise, notamment dans la littérature jeunesse. D'autres auteurs s'efforcent plutôt d'explorer les rapports entre le maître et son chien et d'examiner la charge symbolique de ce dernier qui devient parfois le véritable héros du récit. Ce procédé peut être illustré par différentes œuvres de Guy de Maupassant, notamment son roman Une vie (1883) et des nouvelles comme Pierrot (1883), Histoire d'un chien (1881) et La peur (1882). À la lumière de ces deux tendances, l'originalité de Dumas tient au fait qu'il a recours à ces deux procédés généralement exclusifs et qu'il s'efforce de les entrecroiser.

Dans Les Mohicans de Paris, Roland incarne parfaitement le procédé faisant du chien un compagnon loyal. Alice Kuzniar rappelle que, «traditionnellement, dans les arts, le chien a été associé à la loyauté (dans l'amitié), à la fidélité (dans le mariage), à l'envie (pour l'os que possède l'autre chien), ou à la lascivité (lorsqu'installé sur les jambes de la femme) » (16 ; nous traduisons; voir aussi Kete 24). Dumas reprend donc un trait typiquement associé à la race canine lorsqu'il fait de Roland un adjuvant au héros dans trois des sept récits qui composent l'œuvre. En fait, il constitue une déclinaison de son maître Salvator, avec lequel il partage plusieurs caractéristiques typiques du surhomme du roman populaire: un passé secret, une identité cachée (avant d'être Roland, il s'appelait Brésil) et une vocation à protéger les plus 
faibles. Comme son maître, il est associé à la recherche de la vérité par le biais de la figure du détective ; c'est un limier efficace, capable de repêcher un corps au fond d'un lac et d'user de son flair et de sa mémoire pour retrouver la piste d'un criminel, parfois plusieurs années après le crime (1 100).

Bien qu'il enquête sur des crimes, Salvator ne fait pas l'objet d'une animalisation appuyée comme ce sera fréquemment le cas des détectives dans les récits policiers publiés au cours des années suivantes (Vareille, Filatures, 47-53). Néanmoins, il est en symbiose quasi parfaite avec son chien, témoignant ainsi éloquemment de l'importance du référent animal dans la constitution de la figure de l'enquêteur. Roland sert aussi de prétexte aux personnages et au narrateur pour évoquer l'imaginaire de James Fenimore Cooper, auteur du Dernier des Mohicans (1826) lors de scènes où il permet de retrouver la piste des coupables dans de sombres forêts (Dumas 1 100-1 101). Se dessine l'image d'un personnage canin au croisement d'une représentation bien établie (le fidèle compagnon) et de l'émergence d'une tradition (le limier de récit policier) qui s'imposera quelques années plus tard.

Le second procédé qui cadre la mise en scène des personnages canins, s'avère bien différent. Il est incarné par le chien Babylas qui appartient à la Brocante, une vieille sorcière qui l'idolâtre malgré sa «laideur extraordinaire». Babylas est en effet «petit, trapu, sale au physique, hargneux, grognon, prétentieux au moral » (1 690). Le narrateur décrit longuement les travers de son caractère, expliquant qu'il prend plaisir à ennuyer les autres chiens en faisant ouvrir la fenêtre quand ils ont froid et en la faisant fermer quand ils ont chaud (1 695). La vie de Babylas bascule lorsqu'il tombe amoureux d'une chienne nommée Caramelle. Du jour au lendemain, «[i]l dev[ie]nt doux et bonasse »(1702-1703). Il échange des regards amoureux avec la belle Caramelle mais n'ose s'en approcher parce qu'il est effrayé par son maître. Un jour, il se décide à la poursuivre dans la rue. Une déception l'attend toutefois puisque Caramelle a été entraînée pour séduire les chiens que lui désigne son maître. Dans ce cas précis, il s'agissait d'amener Babylas à suivre Caramelle pour forcer sa maîtresse la Brocante à quitter son appartement pour le rattraper (1 704-1 716), laissant ainsi les lieux déserts.

Cette histoire d'amour canine ne prend son sens qu'à la lumière de ce dernier revirement. Elle semble d'abord accessoire aux intrigues principales, présentant un chien comme le héros de trois chapitres d'un roman bien avancé (chapitres CCXXI, CCXXII et CCXXIII) dans lequel il n'a jamais été fait mention auparavant de ce personnage. Le premier chapitre présentant Babylas 
s'intitule d'ailleurs : «Où l'auteur demande pardon au lecteur de lui faire faire connaissance avec un nouveau personnage ». Cette fugue amoureuse joue cependant un rôle important dans le déroulement des péripéties : elle mène à l'élucidation d'un meurtre, à un chantage, à un vol et à un enlèvement. Malgré ce cadrage policier, cet «intermède fantaisiste de délassement» (Anselmini 288) impliquant Babylas et Caramelle rappelle plutôt un conte ou une fable dont il faut tirer certaines leçons, notamment que l'amour remet Babylas sur le droit chemin et que l'éducation peut pervertir « les meilleurs caractères » (Dumas 1 693). L'univers de la fable est un intertexte important dans ce roman où divers chapitres reprennent des titres de La Fontaine (« Le chêne et le roseau », 318) ou s'en inspirent («Le lierre et l'ormeau », 994) ${ }^{1}$. D'ailleurs, l'œuvre se conclut sur une «Moralité ». On ne peut cependant réduire la mise en scène de ces chiens à cet intertexte.

\section{Rendre les chiens lisibles et parlants}

L'anthropomorphisation des personnages canins des Mohicans de Paris est fondée sur deux procédés récurrents : leur association à des types connus des lecteurs et le recours au motif de la communication. Dumas emploie le premier de façon presque systématique : même les chiens qui ne sont que rapidement évoqués sont associés à des types au moyen d'une catégorisation ironique pastichant des disciplines comme la phrénologie et la physiognomonie pour présenter le bouledogue comme colérique et belliqueux, le lévrier comme craintif et tremblant et ainsi de suite (1 694). Ce procédé est déployé de façon encore plus appuyée dans le cas des personnages canins qui jouent un rôle significatif dans l'intrigue.

On l'a déjà deviné, Roland correspond au type romanesque du fidèle acolyte, abondamment employé dans le roman populaire. Parmi les plus connus, retenons le Chourineur dans Les mystères de Paris (1842-1843) de Sue, Passe-Partout dans Le tour du monde en quatrevingts jours (1872) de Jules Verne, Planchet dans Les trois mousquetaires (1844) de Dumas. Ces «acolytes »sont fréquemment associés à des personnages canins en raison de leur fidélité. Le Chourineur dit à Rodolphe : «Je me sens comme votre bouledogue » (Sue 150), transformation canine longuement analysée (non sans ironie et sans agacement) par Karl Marx dans La sainte famille (196-199)². En attribuant un tel statut à Roland, le récit le place sur un pied d'égalité avec les autres personnages qui font preuve du même dévouement aveugle pour le héros Salvator. Ce chien se joint de ce fait à une galerie d'hommes du peuple, d'artistes, d'honnêtes femmes et 
d'hommes respectables, nobles ou exerçant des professions telles que médecins, financiers ou militaires. Parmi eux, Roland s'avère particulièrement utile : il permet d'élucider un meurtre, une tentative de meurtre et un enlèvement et il sauve deux personnages différents en plus d'en arrêter un troisième. C'est dire que tant dans le portrait louangeur dont il fait l'objet que dans son « utilité » au sein du déroulement des péripéties, Roland l'acolyte fidèle, fait très bonne figure.

Pour sa part, Babylas, en raison de son caractère déplaisant, est décrit comme « résuma[nt] à lui seul tous les vices d'un vieux garçon » (1690). C'est sous ce parrainage que le texte place ses comportements désagréables jusqu'au moment où, après avoir posé les yeux sur la belle Caramelle, il devient un amoureux transi. Dumas lui fait éprouver tous les tourments romantiques de la passion, comme une perte d'appétit, des palpitations et des nuits d'insomnie ou peuplées de rêves amoureux : «Ce fut à la fois pour Babylas une nuit de tortures inconnues et de délices inouïs : [...] il vit passer [...] les ombres de tous les chiens qui avaient aimé, et de toutes les Hélènes et de toutes les Stratonices à quatre pattes qui avaient produit des passions insensées » (1 697). Devant l'apparente insensibilité de l'élue de son cœur, il dépérit à vue d'œil mais lorsqu'il croit être aimé, il change et délaisse ses manies détestables (l'amour le rend meilleur). Lui sont ainsi attribués différents traits banals des amoureux romantiques littéraires et théâtraux de l'époque. Le caractère grandiloquent et dramatique de cette mise en scène ajoute une touche d'humour au récit tout en cadrant l'anthropomorphisation de Babylas dans un contexte bien connu du lecteur.

Caramelle, la belle chienne manipulatrice, fait l'objet d'un travail analogue. Son attitude respecte les convenances de la bonne société : «obligée de subir [1]e regard de feu [de Babylas, elle] baiss[e] timidement les yeux, et pass[e] sans détourner la tête » (1 696). De cette «timidité », son amoureux déduit qu'elle est «honnête et belle ». Selon le narrateur, il paraphrase une réplique de Hamlet - «Sage et belle »- et illustre que, « en circonstance pareille, pareille impression se produit sur l'homme et sur l'animal, sur le prince et sur le chien » (1 696). La déduction de Babylas est toutefois erronée : manipulatrice et séductrice, Caramelle est plutôt ce qu'il convient d'appeler une «chienne fatale». Après avoir suscité une passion presque mortelle chez Babylas, elle lui fait perdre tout sens commun et le rend si amoureux qu'il sacrifie tout pour elle. Si le narrateur reste silencieux sur les pensées de Caramelle - contrairement à Babylas -, le motif de la femme qui séduit un homme et le rend «malade d'amour» pour s'approprier ses biens apparaît clairement au lecteur. Non seulement il est abondamment utilisé 
au théâtre et dans la littérature de l'époque mais Les Mohicans de Paris comporte le récit détaillé de l'histoire d'une telle séductrice criminelle. Patent et parfaitement assumé, ce procédé consistant à croiser personnages canins et types, sociaux et littéraires, est renforcé parce que ces chiens communiquent parfaitement entre eux, second procédé d'anthropomorphisation employé par Dumas.

Les passages consacrés à nos trois personnages canins sont tous chapeautés par le motif de la communication. Le geste n'est pas anodin puisqu'il a souvent été posé que « ce qui sépare l'humain de la bête est le mutisme de cette dernière »(Kuzniar 25 ; nous traduisons). Prenant résolument le contre-pied de cette perspective, le narrateur des Mohicans de Paris décrit une compréhension mutuelle du chien et du maître lors de leurs enquêtes communes. Non seulement le premier obéit aux ordres du second mais il répond à ses questions :

- $\quad$ Eh bien, [Roland,] trouve le moyen, toi, puisque tu te fâches [dit Salvator]. J'attends [...].

[Roland] semblait ne pas perdre un mot de ce que disait son maître. Aussi, ne pouvant, à lui tout seul, appliquer le moyen, se contenta-t-il de l'indiquer [...].

- $\quad$ Tu es la suprême sagesse, mon cher [...], dit Salvator, et tu as parfaitement raison. (1 094-1 095)

De tels échanges sont récurrents : «Mais [Roland] secoua la tête comme pour répondre qu'il était bien inutile de chercher»(1948), «Le chien le regarda et sembla dire : “"Mais vois donc toi-même"” » (1 101), «Le chien[,] regardant son maître comme pour lui dire : “À ton tour"” » (2 168). Malgré une modalisation systématique de la part du narrateur, l'impression qui se dégage ici, est celle d'une compréhension parfaite. Au-delà des qualités de fidélité et d'intelligence, ce qui rend ce chien exceptionnel, est sa capacité à communiquer des émotions et des idées complexes : «[L]e chien dumasien est le plus éloquent des animaux : il possède la parole, il est un «chien parlant » langue faite corps, âme en mouvement, pensée lisible à même ses membres [; son langage] est susceptible de faire entendre les plus fines nuances » (Grivel 243). La contribution de Roland aux enquêtes menées par Salvator illustre cette «langue faite corps ». Un autre passage s'avère aussi éclairant : s'adressant à Roland, « Salvator 1'avait retenu, et, chose singulière ! plutôt avec le raisonnement, comme s'il eût retenu un homme, qu'avec un ordre, un commandement, un juron, comme on retient un chien » (1080). Révélateur du caractère posé de Salvator, qui use toujours avec parcimonie du pouvoir qu'il exerce sur les autres personnages, ce passage pose un phénomène crucial sur lequel nous reviendrons : le 
brouillage de ce qui sépare le chien et l'humain. Pour l'instant, soulignons que le motif de la communication est tout aussi présent à propos de Babylas et Caramelle. La communication «corporelle » est toutefois remplacée ici par la faculté pour les personnages canins de parler entre eux.

L'épisode dont Babylas et Caramelle sont les principaux acteurs, est introduit au moyen d'un dialogue entre le narrateur et le lecteur. Ce dernier s'insurge que le premier « cherche à [...] faire accroire que les chiens parlent, haussent les épaules, sourient » (1 690). Le narrateur défend une à une chacune de ces affirmations au moyen d'une série d'exemples et conclut en disant que ce n'est pas parce que les humains ne comprennent pas les chiens que ceux-ci ne parlent pas (1 691-1 692). Le narrateur tente de vaincre les éventuelles réticences du lecteur face à cette prosopopée qui est par ailleurs propre à ce seul épisode. Les aventures de Babylas mettent ainsi en scène un groupe de chiens qui discutent fréquemment entre eux, de façon tout aussi claire que soignée : «[Babylas] s'avança vers ses camarades, leur fit de franches excuses, leur demanda loyalement pardon de ses torts, et, après cette amende honorable, les supplia de lui rendre leur amitié, leur promettant sur l'honneur d'en observer les règles les plus difficiles, d'en accomplir les devoirs les plus rigoureux »(1 702-1 703). Le caractère pompeux de ces excuses par un personnage à quatre pattes fait sourire mais ses camarades ne sont pas en reste : ils multiplient les conciliabules soumis aux règles d'une politesse exemplaire (1 694 ; 1699). Ils proposent aussi des versions modifiées des proverbes humains : « un temps à ne pas mettre un chien à la porte » devient «un temps à ne pas mettre un homme à la porte» (1 698). L'ampleur du phénomène laisse percevoir que l'attribution de la parole aux personnages canins n'est pas accessoire : il s'agit d'un aspect essentiel de leur mise en scène et même d'un rouage important de l'architecture narrative du roman.

\section{Instrumentalisations et politisation du chien}

L'association des personnages canins à des types bien connus sert, dans Les Mohicans de Paris, de raccourci narratif permettant l'économie de longues descriptions. Plusieurs des passages mettant en scène des chiens, au premier chef la vignette satirique de Babylas et Caramelle, ont aussi pour but d'offrir des pauses au sein du récit des péripéties. L'anthropomorphisation dépasse cependant ces considérations. Rappelons que la «question animale » a une réelle actualité lorsque Dumas rédige son roman et qu'elle est associée à 
plusieurs débats et enjeux importants (Agulhon; Estève; Pierre). Par exemple, certains observateurs du dix-neuvième siècle, insistant sur le paradigme de la lutte du faible contre le fort, rapprochent les efforts pour la protection des animaux des luttes pour les droits des femmes, ce qui justifierait l'intérêt de ces dernières pour les causes animales (Kete 8). Plus connu encore est le rapprochement avec les débats accompagnant les revendications des classes populaires. Dans le vibrant plaidoyer pour celles-ci qu'est Le peuple, Michelet emploie l'expression « frère inférieur » afin d'unir les violences dont sont victimes les classes populaires et le règne animal. Le geste ne fait pas l'unanimité, comme le soulignent différents comptes rendus de l'ouvrage offerts dans des journaux, parce que «tout un courant de l'opinion populaire voyait dans la zoophilie une concurrente, et non une propédeutique, pour la philanthropie » (Agulhon 92). En d'autres mots, l'énergie consacrée à protéger les animaux serait détournée des efforts destinés à améliorer la situation du peuple.

La question animale comporte aussi de nombreuses ramifications. Par exemple, du point de vue de plusieurs fonctionnaires parisiens, elle s'articule aux travaux visant à moderniser la capitale : «Le but de la protection des animaux était initialement de façonner le Paris populaire selon les normes de l'Haussmannisation : utilité, prédictibilité, et contrôlabilité » (Kete 21). Plus significative encore est sa participation aux débats portant sur la place de la religion en France puisque l'épineuse question de la séparation entre l'animal et l'humain divise les avis. Les théories du grand architecte de la prison panoptique Jeremy Bentham (1748-1832) sur la souffrance des animaux publiées en Angleterre en 1789 ont ranimé le débat, tout comme, plus récemment, la création de la Société de Protection des Animaux en 1845 et différents ouvrages de l'époque. Par exemple, lorsque Michelet fait de l'animal un «frère inférieur », il pose entre l'homme et la bête une distance faite de «degrés » au lieu de la «coupure sacrée » prônée par les autorités religieuses (Agulhon 94). Les droits des animaux ont aussi été instrumentalisés pour remettre en question les dogmes religieux. Ainsi, Michelet s'est interrogé sur la notion de péché originel à partir de la situation de l'enfant n'ayant pas reçu le baptême mais aussi à partir de celle de la bête (Fontenay 618). L'auteur de l'Histoire de France, dont l'anticléricalisme a souvent été relevé (Pelckmans), veut mettre en lumière certaines failles du discours religieux. La question animale prend ainsi une vaste portée dans l'imaginaire de l'époque.

C'est dans ce contexte que doit être recadré ce travail de Dumas consistant à attribuer à ses personnages canins des traits qui les humanisent. En soulignant leur « humanité », plutôt que 
leur animalité, le roman institue ce brouillage des frontières que nous avons déjà évoqué. Celuici, fréquent dans les fables, les contes et les caricatures, prend ici une saveur particulière puisque Les Mohicans de Paris est une œuvre de fiction affirmant décrire de façon exacte « ce qu'était le Paris physique et moral des dernières années de la Restauration » (9). Y surgissent plusieurs chapitres interrompant le récit pour peindre le contexte sociopolitique de l'époque (chapitres I, CVII, CVIII, CLIII). L'irruption d'animaux doués de la parole jure avec l'ensemble de l'œuvre, plutôt marquée par un romantisme à saveur réaliste. Néanmoins, il apparaît clairement que ces personnages canins «forment système» (Grivel 260) afin de redoubler le projet politique du roman.

Tout en revisitant le romantisme et ses idéaux, le narrateur des Mohicans de Paris critique la Restauration mais aussi le Second Empire. Ce roman-feuilleton est construit comme un instrument d'éducation et de persuasion qui offre notamment une mise en scène du peuple militante et nuancée. La représentation des personnages canins humanisés participe à ce portrait de la société dans cette «fable politique » (Dumasy 199) qui tente de conjuguer le passé et le présent :

Mais il ne s'agit pas pour Dumas de convoquer le passé politique et littéraire - le sien et celui de sa génération - à des fins d'immobilisme politique et esthétique : il s'agit, sans le renier, de le dépasser à partir du point de vue, rétrospectif, d'un écrivain situé dans un autre moment littéraire et socio-politique : Les Mohicans de Paris traduisent ainsi une évolution à la fois de l'esthétique et de la position politique de leur auteur (199).

La prétention à l'exactitude de cette réécriture actualisée doit ainsi être envisagée avec précaution. Lise Dumasy souligne par exemple que le personnage de Jean Robert est ce que Dumas «aimerait maintenant avoir été » (204). En fait, le roman présente un véritable optimisme. Tout en proposant ce qui aurait pu (ou aurait dû) être, l'œuvre offre du même souffle un aperçu de ce qui, malgré le passage du temps, pourrait encore être, intégrant un véritable projet de société à cette reconstruction historique.

C'est ainsi que le souhait révolutionnaire qui anime les personnages valorisés par la narration, se confond avec les valeurs républicaines si chères à Dumas (Schopp, Dumas, 449). L'extraordinaire mobilité sociale des personnages des Mohicans de Paris en est une illustration : s'y multiplient les rencontres qui font fi des divisions sociales au profit de valeurs comme l'intelligence et la charité. Idéalisant ses jeunes héros, le narrateur souligne leur capacité et leur désir de se mobiliser pour des causes sociales ou politiques. La communauté s'y impose comme 
une force puissante : l'unité des quatre héros, tous des artistes, et celle des quatre héroïnes, issues de toutes les classes sociales mais réunies par leurs valeurs, n'ont rien à envier à celle des mousquetaires de Dumas. La fameuse devise de ces derniers, «Tous pour un, un pour tous » (Mousquetaires, 131), leur convient parfaitement. Dans ce roman cumulant des projets politiques, esthétiques et biographiques (Dumasy 202), tout fait système, incluant la question de ce qui sépare l'homme de la bête.

Le narrateur des Mohicans de Paris attribue ainsi aux chiens tout autant les qualités valorisées dans l'œuvre (intelligence, loyauté, tolérance) que les comportements décriés et associés à la civilisation (prétention, manipulation). Plutôt qu'une opposition essentialiste entre chien et humain, se dessine entre eux une analogie et même un horizon commun de possibilités. Ainsi, Roland fait preuve des plus belles qualités morales que l'on observe chez les autres acolytes humains de Salvator, et témoigne de multiples façons de ce que la noblesse n'est pas qu'une question de naissance.

Quant à lui, Babylas fait l'objet d'un chapitre intitulé : «Où l'on verra que la mauvaise éducation peut gâter les meilleurs caractères » (1 693). Le narrateur explique ainsi comment, suite aux louanges de sa maîtresse, il en est venu à «s'imaginer qu'il était le plus joli, le plus beau, le plus spirituel, le plus aimable, le plus séduisant des chiens » (1 690), bien qu'il soit l'exacte antithèse de ce portrait. Ce processus de corruption par l'éducation n'est pas associé ici à une classe sociale en particulier, bien au contraire. À la lumière des différents épisodes du roman, l'histoire de Babylas peut évoquer une aristocratie ayant perdu tout contact avec la réalité et ayant une foi aveugle dans les louanges dont elle fait l'objet. Le sujet est d'actualité sous le Second Empire et Dumas fustige à de multiples reprises l'aristocratie figée dans le temps, celle de la seconde Restauration comme celle entourant Napoléon III. Cependant, à la lumière des origines modestes de Babylas, de son milieu de vie et de son portrait par le narrateur, on peut également voir dans ce passage une évocation d'un autre thème récurrent du roman: l'importance d'une éducation éclairée des classes populaires, autre sujet lié à l'actualité, tant au moment de l'écriture du roman qu'à la période où se déroule l'intrigue.

C'est dire que l'histoire de Babylas et celle de Roland ne peuvent être perçues comme des recréations simplistes de la société au sein du monde animal qui associeraient certaines espèces ou certains animaux à des classes sociales précises. Le brouillage entre l'homme et la bête porte plutôt sur la bestialité du premier, sur l'humanité de la seconde et sur le fait que tous peuvent 
poser les meilleurs et les pires gestes. L'idéal républicain surgit ici en filigrane, marquant une visée allégorique dans la mise en scène des personnages canins et soulignant que cette dernière n'a rien d'une digression sans conséquence. Grivel l'a souligné avec beaucoup de justesse : «Dumas n'est jamais autant sérieux que quand il ne le paraît pas » (55).

\section{Conclusion}

Dans le vaste « océan d'encre » (Schopp, «Paris », 2 655) des Mohicans de Paris, les personnages canins sont très présents et participent intimement aux enjeux essentiels du roman. Leur mise en scène repose sur l'association à différents types et sur la capacité à communiquer, voire à parler. Rappelant différents grands modèles, à commencer par La Fontaine dont l'ombre est ici omniprésente, une telle fictionnalisation s'avère pourtant atypique par rapport aux romans comparables de l'époque. Dans Les Mohicans de Paris, l'anthropomorphisation des bêtes s'appuie sur la coexistence du conte, de la fable et de la satire. Elle est parfois poussée, avec une touche d'humour, jusqu'au renversement. Les colocataires de Babylas se disent : «Ne soyons pas impitoyables pour un des nôtres : nous sommes des chiens, et non des hommes » (1 699). Si ce renversement à vocation comique n'est pas généralisé, le brouillage des limites entre l'homme et la bête ne surprend pas chez un auteur qui aimait à dire que «[1]es chiens sont des candidats à l'humanité »(Schopp, Dictionnaire, 113) et qui, conséquemment, leur accorde ici un statut particulier.

À cette anthropomorphisation se greffe parfois une animalisation des humains, par exemple avec le portrait de différents personnages, dont celui de Jackal que nous avons évoqué plus haut. Le roman de Dumas est marqué par un désir de souligner ce qu'il peut y avoir d'animal dans l'être humain et spécifiquement dans son comportement ${ }^{3}$. Étudiant diverses représentations artistiques du chien, Kuzniar, évoque ces écrivains qui «compliquent [et] 'délinéarisent' la ligne entre personnages canins et humains » (6). Le Dumas des Mohicans de Paris correspond parfaitement à cette description. En fait, la formule «délinéarisation » rend justice à toute la poétique romanesque de cet auteur. Les Mohicans de Paris en est un exemple éloquent puisque Dumas y privilégie la multiplication des récits autour de différents centres de gravité, au nombre desquels se trouvent l'humanité des animaux et l'animalité des hommes. 


\section{Bibliographie}

Agulhon, Maurice. «Le sang des bêtes. Le problème de la protection des animaux en France au dix-neuvième siècle ». Romantisme 31 (1981) : 81-109.

Anselmini, Julie. Le roman d'Alexandre Dumas père ou La Réinvention du merveilleux. Genève : Droz, coll. Histoire des idées et critique littéraire, 2010.

Dumas, Alexandre. Les Mohicans de Paris. Paris : Gallimard, coll. Quarto, 1998 [1854-1859].

---. Les trois mousquetaires, Paris : Garnier-Flammarion, coll. GF, 1975.

Dumasy, Lise. «Les Mohicans de Paris ou comment être Romantique sous le Second Empire ». Entre presse et littérature: Le mousquetaire, journal de M. Alexandre Dumas (18531857). Dir. Pascal Durand et Sarah Mombert. Genève : Droz, coll. Bibliothèque de la Faculté de Philosophie et Lettres de l'U de Liège, 2009. 197-228.

Estève, Christian. «La 'question' canine en France au milieu du dix-neuvième siècle ». L'animal en politique. Dir. Paul Bacot, Eric Baratay, Denis Barbet, Olivier Faure et Jean-Luc Mayaud. Paris : Harmattan, coll. Logiques politiques, 2003. 83-99.

Fontenay, Elisabeth. Le silence des bêtes. La philosophie à l'épreuve de la bestialité. Paris: Fayard, 1998.

Grivel, Charles. Alexandre Dumas, l'homme 100 têtes. Villeneuve-d'Ascq : PU du Septentrion, coll. Objet », 2008.

Kalifa, Dominique. Crime et culture au dix-neuvième siècle. Paris : Perrin, coll. Pour l'histoire, 2005.

Kete, Kathleen. The Beast in the Boudoir : Petkeeping in Nineteenth-Century Paris. Berkeley : U of California P, 1994.

Kuzniar, Alice, A. Melancholia's Dog. Chicago : The U of Chicago P, 2006.

Marx, Karl. La sainte-famille. Paris : Éditions sociales, 1972.

Michelet, Jules. Le peuple. Paris : Éditions d'aujourd'hui, coll. Les introuvables, 1977.

Pelckmans, Paul. « 'Le Prêtre, la Femme, la Famille' : notes sur l'anticléricalisme de Michelet ». Romantisme 23 (1979) : 17-30.

Pierre, Éric. «La zoophilie dans ses rapports à la philanthropie, en France, au dix-neuvième siècle ». Cahiers d'Histoire 3-4 (1997) : 655-675.

Schopp, Claude. Alexandre Dumas : «le génie de la vie ». Paris : Fayard, 1997. 
---. «Paris ne nous appartient plus ». Les Mohicans de Paris. Alexandre Dumas. Paris : Gallimard, coll. Quarto, 1998 [1854-1859] : 2 655-2 721.

---. Dictionnaire Dumas. Paris : CNRS, 2010.

Sue, Eugène. Les mystères de Paris. Paris : Robert Laffont, coll. Bouquins, 1989.

Vareille, Jean-Claude. Filatures : itinéraire à travers les cycles de Lupin et Rouletabille. Grenoble : PU Grenoble, 1980.

---. « Préhistoire du roman policier ». Romantisme 53 (1986) : 23-36.

---. L’homme masqué, le justicier et le détective. Lyon : PU Lyon, coll. Littérature et idéologies, 1989.

\section{NOTES}

${ }^{1}$ Notons qu'il s'agit ici de titres évoquant des fables de La Fontaine qui mettent en scène des végétaux, lesquelles sont bien moins nombreuses que les fables présentant des animaux. Ces dernières auraient d'ailleurs, peut-être, constitué un intertexte plus attendu dans ce roman.

${ }^{2}$ Sur ce personnage du Chourineur nous renvoyons à l'article de Nigel Lezama «Le bestiaire au dix-neuvième siècle : animalité, pauvreté et criminalité dans Les mystères de Paris ", publié dans ce même numéro de Voix plurielles et qui en propose une analyse fouillée.

${ }^{3}$ Rappelons que l'animalisation des humains ne concerne pas strictement des personnages mis à l'écart par leurs vices ou leurs gestes. Ainsi, bien que le phénomène ne soit pas très appuyé, Salvator est animalisé lorsqu'il enquête sur un crime dans un vaste parc (et il est, nous l'avons souligné, en symbiose avec son chien). 